FINAL REPORT

U.S. Department of Energy

\title{
Characterization of Chemically Modified Enzymes for Bioremediation Reactions
}

\author{
Principal Investigator: Brian H. Davison \\ Institution: Oak Ridge National Laboratory
}

Collaborator: Dr. M. W. W. Adams, The Univers ity of Georgia, Athens, GA

Project Number: $\mathbf{5 5 0 3 3}$

Report Date: September 22, 2000

Grant Number: DE-AC05-00OR22725

Grant Project Officer: Robert E. Price

Project Duration: October 1, 1996 to August 31, 2000 


\section{TABLE OF CONTENTS}

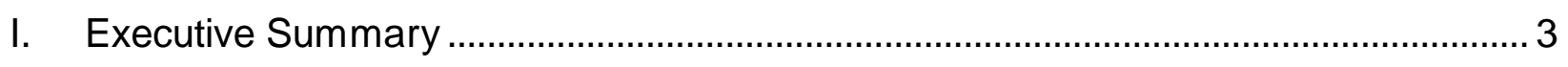

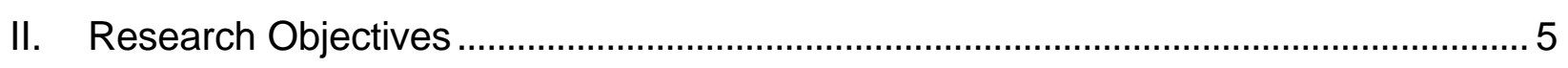

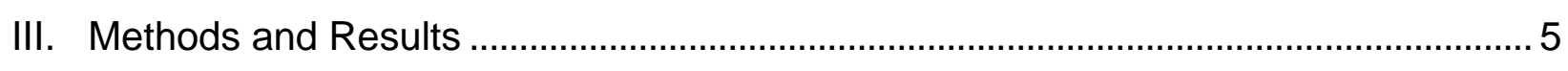

IV. Relevance, Impact and Technology Transfer ....................................................... 8

V. Project Productivity ..................................................................................... 10

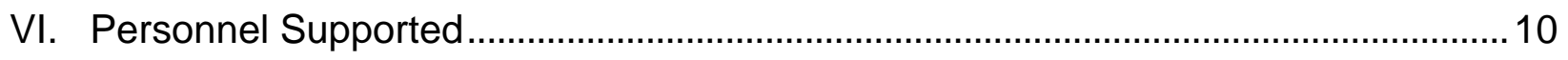

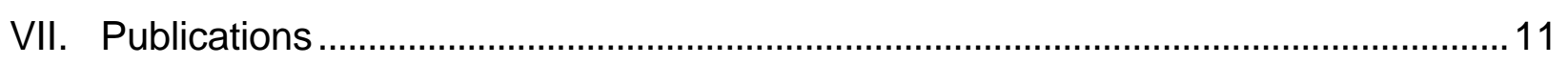

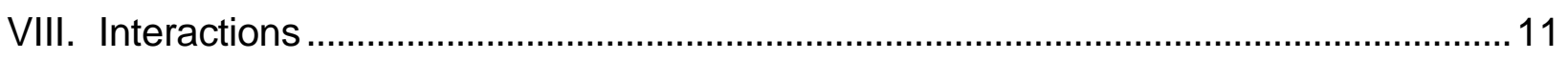

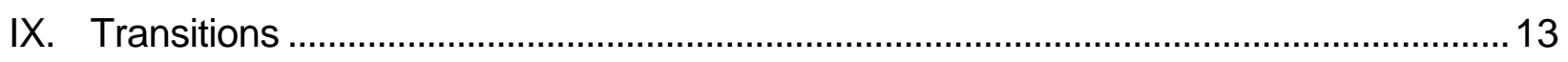

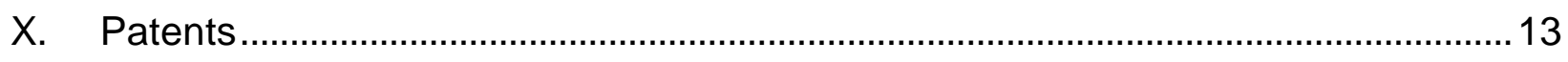

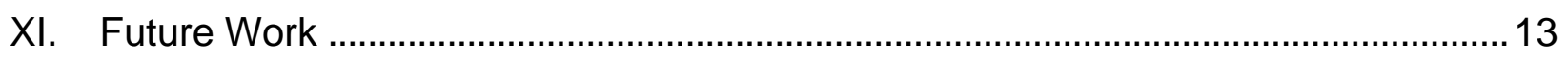

XII. Literature Cited .................................................................................. 14

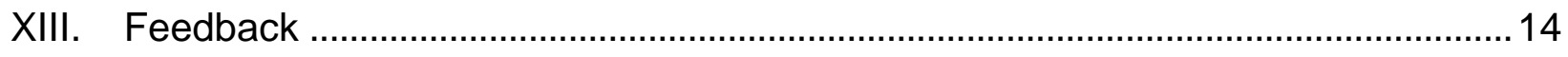

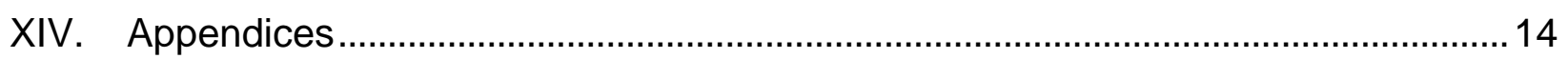




\section{Executive Summary}

Remediation processes frequently involve species possessing limited solubility in water. We have explored novel strategies that use molecularly modified redox enzymes with enhanced activity and stability for the remediation of recalcitrant compounds in organic solvents. The performance of naturally occurring enzymes is usually quite limited in such organic environments. The primary objective of the current work is to gain a fundamental understanding of the molecular and catalytic properties of enzymes that have been chemically modified so that they are catalytically active and chemically stable in organic solvents. The study also sought to develop and characterize oxidative and reductive enzymes that are necessary to achieve these dechlorinations. The premise for this study is that stabilized and activated enzymes, which can function at hash chemical conditions, are optimally suited for bioremediation in nonaqueous media where substrates of interest are more soluble and processed with greater efficiency. This unique strategy is examined with respect to the degradation of chlorophenols and PCBs which may persist in organic DNAPLs.

This report summarizes our three-and-a-half year effort. We have obtained promising results both to demonstrate the proposed remediation strategy and to reveal fundamentals of enzymatic catalysis in organic media. Enzyme modification was demonstrated to allow redox enzymes to be more active in organic solvents for dechlorination. In one effort, ligninase (LiP) from Phanerochaete chrysosporium was modified with polyethylene glycol (PEG); this significantly enhanced enzymatic degradation of chlorophenol and pentachlorophenol (PCP) in water-solvent mixtures. In another effort, basic catalytic behaviors of the chemically modified 
hyperthermophilic redox enzymes including ferredoxin, hydrogenase, and aldehyde oxidoreductase were examined in organic solvents. In a more directed effort to attack PCBs directly using single oxidative or reductive enzymes (e.g., liginase and hydrogenase), the results were inconclusive. This is likely due to the insufficient redox potential of these single enzyme systems.

In summary:

\section{A. Remediation of chloroorganics by modified enzymes}

- Ligninase (LiP) was modified by poly(ethylene glycol) (PEG) and examined for the degradation of pentachlorophenol (PCP) in water-solvent mixtures. It was demonstrated that the PEG-LiP is 11-fold more efficient than native LiP for PCP degradation.

- A protocol for PCB analysis was developed to minimize losses and errors while looking for PCB attack by oxidative enzymes. Reproducibilities of about $30 \%$ were obtained overall and for individual Aroclor fingerprint peak analysis.

- $\quad$ PCB Aroclor 1242 and hexachlorobiphenyl were tested for degradation by ligninase, PEGligninase, and Cytochrome $\mathrm{C}$ in the presence of $10 \% \mathrm{MeCN}$ and $0.1 \mathrm{mM} \mathrm{H} 2 \mathrm{O} 2$. We also tested hyperthermophile extracts from Pyrococcus furiosus, Thermotoga maritima, and Pyrobaculum aerophilum. Within experimental error, no evident reaction was observed.

\section{B. Oxidative enzyme catalysis in organic media}

- Basic catalytic behaviors of the chemically modified hyperthermophilic metalloenzymes of $P$. furiosus including ferredoxin, hydrogenase and aldehyde oxidoreductase were examined in organic solvents. Electron transfer is the key to redox biodegradation.

- Modified PEG-ferrodoxin showed better stability in organic solvents with the same redox potential of the native enzyme. PEG-fd maintained its active site iron-sulfur cluster in DMSO and acetonitrile despite a significantly lower redox potential, but it was more susceptible to oxidation and thermal denaturation than the native protein.

- PEG-hydrogenase is fully soluble and active in organic solvents.

- Two new hyperthermophilic hydrogenases were isolated. 


\section{Research Objectives}

Remediation processes frequently involve species possessing limited solubility in water. For this project, we were interested in novel strategies using molecularly modified enzymes with enhanced activity and stability for remediation of recalcitrant compounds in organic solvents. Performance of naturally occurring enzymes is usually quite limited in such organic environments. The primary objective of the work was to gain a fundamental understanding of the molecular and catalytic properties of enzymes that have been chemically modified so that they are catalytically active and chemically stable in organic solvents. The premise was that stabilized and activated enzymes, which can function under harsh chemical conditions, are optimally suited for bioremediation in nonaqueous media where substrates of interest are more soluble and processed with greater efficiency. This unique strategy was examined with respect to the degradation of chlorophenols and PCBs.

\section{Methods and Results}

\section{A. PEG Modification of LiP and Associated Bioremediation Applications}

LiP was modified using PEG to enhance its activity and stability for the biodegradation of PCP in the presence of acetonitrile $(\mathrm{MeCN})$, an organic solvent. MALDI-TOF mass spectrometry analysis showed that the modified enzyme had one or two PEG groups attached to each protein molecule. The modified enzyme retained $100 \%$ of its activity in aqueous solutions and showed enhanced activity in the organic solvent. Activity of the modified enzyme was found to be over twice that of the native enzyme in the presence of $10 \%(\mathrm{v} / \mathrm{v}) \mathrm{MeCN}$. The solubility of PCP was enhanced significantly by the addition of $\mathrm{MeCN}$ to aqueous solutions. Capitalizing on the enhanced substrate solubility and the increased activity of the modified enzyme, $\underline{\text { catalytic }}$ efficiency of the modified LiP in solutions containing $15 \% \mathrm{MeCN}$ was over 11-fold higher than that of the native enzyme in aqueous solutions (from 44 to $480 \mathrm{~mol} \mathrm{PCP} / \mathrm{mol} \mathrm{LiP} \cdot \mathrm{h}$ ).

Tetrachlorobenzoquinone (TCBQ) was the only degradation product observed that had been previously reported in the literature. Our work revealed formation of three additional intermediates, which contributed to closing the mass balance between the yield of TCBQ and the disappearance of PCP. Furthermore, an additional product peak was observed by HPLC when PEG-LiP, instead of the native enzyme, was used. This result implies that the chemical modification of enzymes may affect the reaction specificity.

\section{B. Fundamentals of Modified Enzymes in Organic Solvents}

One aspect of this research focused on the basic properties of a hyperthermophilic redox protein, ferredoxin ( $\mathrm{Fd}$ ) fom Pyrococcus furiosus, in organic solvents. This study contributed to our knowledge of oxidation and reduction chemistry of biocatalytic systems placed in nonaqueous reaction media. 
Thermal stability: Studies were carried out both with native and with PEG-modified Fd. Both enzymes dissolve in very polar organic solvents (e.g., DMSO) whereas PEG-Fd was also soluble in much less polar solvents including ethanol, propanol, butanol, benzene, and toluene. Although thermal stabilities of both native and PEG-Fd were lower in DMSO compared to that in water ( $\mathrm{t}_{1 / 2}$ at $80^{\circ} \mathrm{C}$ of 60 and $<10 \mathrm{~min}$, respectively), PEG-Fd showed better stability in organic solvents (the $\mathrm{t}_{1 / 2}$ at $80^{\circ} \mathrm{C}$ increased to $30 \mathrm{~min}$ in toluene).

Properties of the iron-sulfur cluster in chemically-modified ferredoxin: Stability and redox properties of the $[4 \mathrm{Fe}-4 \mathrm{~S}]$ cluster in a chemically-modified ferredoxin were investigated in a range of solvents and at a range of temperatures. PEG-modified ferredoxin maintains its ironsulfur cluster in DMSO and acetonitrile, as well as in aqueous media, but it became more susceptible to oxidation and thermal denaturation than the native protein. This was the first demonstration of superoxidized states $(3+/ 2+)$ of a $4 \mathrm{Fe}$-cluster which were accessible by direct electrochemistry in DMSO. This is significant to EM problems as electron transfer reactions are key to the majority of useful biotransformations of biotechnological significance. This is the first study to address the properties of an iron-sulfur center (which is the most ubiquitous electron carrier in nature) in organic media while still within a protein environment. Such information would be extremely useful in the future use and/or design of oxidoreductase-type enzymes in solvents.

The redox potentials of the native and PEG-Fds were determined using cyclic voltammetry. In general, little difference in redox potential was observed between the native and modified enzyme, either in aqueous solution or in solvents such as DMSO. This indicated that the integrity of the iron-sulfur cluster was not altered by PEG modification. However, organic solvent appeared to be capable of dramatically altering the redox potential of the protein. The redox potential of Fd was measured to be $-690 \mathrm{mV}$ in DMSO, which is much lower compared to that in aqueous solution $(-390 \mathrm{mV}$ at $\mathrm{pH} 8)$. Since the absorption spectra of the protein remained intact in DMSO, the dramatic shift in redox potential is most likely due to the changes in cluster environment rather than structural variations. This was verified using a mutant Fd, D14S, in which a cluster-ligating aspartate is replaced by serine. The ability to change cluster potential may prove useful in allowing the kinetics of electron transfer during catalysis to be altered depending on the choice of solvent.

Protein interactions in organic solvents: The effect of the PEG modification on the ability of Fd to accept electrons in organic solvents from another enzyme, aldehyde ferredoxin oxidoreductase (AOR), was investigated. The information gathered will be helpful in designing multi-enzyme redox systems to degrade recalcitrant organic pollutants. AOR oxidizes aldehydes to acids and directly reduces Fd. While there was no activity in aqueous solution when either one of the two proteins was modified with PEG (PEG-AOR or PEG-Fd), remarkably, a high rate of catalysis ( $50 \%$ the activity of the native proteins) was observed when both proteins were modified and used together. No activity was detected with the two PEG-proteins in toluene or ethanol.

Sulfur reduction by solvent-soluble, chemically modified hydrogenase: It was shown that hydrogenase taken from Pyrococcus furiosus was completely soluble in organic solvents when it was modified with polyethylene glycol. Under such conditions, the enzyme catalyzed the 
reduction of elemental sulfur to hydrogen sulfide using hydrogen gas as the electron donor. However, water was also required for the reaction as the source of protons.

Other new hydrogenases of $P$. furiosus were purified and characterized: a) a second hydrogenase (II) with properties closely resembled those of the hydrogenase (I) previously purified from this organism, with the exception of its sulfur-reducing properties. Hydrogenase II has a much higher affinity for both elemental sulfur and polysulfide than does hydrogenase I; and b) $\underline{a}$ membrane-bound hydrogenase - It has very high $\mathrm{H}_{2}$ evolution activity but did not, surprisingly, catalyze the reduction of elemental sulfur to $\mathrm{H}_{2} \mathrm{~S}$. This is the first membrane-bound enzyme to be purified from this organism.

\section{Enzymatic degradation of PCBs:}

We developed a protocol in order to to measure PCB degradation by various enzymes and modified enzymes using GC-ECD analysis of PCBs. Initial tests found that the PCB concentration in water-acetonitrile mixtures decreased with time. However, adsorption of PCBs to the wall, cap, and maybe pipette tips, was suspected to be the cause. A surfactant, Witconal SN 70 (at a concentration of $0.1 \sim 0.5 \% \mathrm{v} / \mathrm{v}$ ), was used to eliminate most of the sorption. Both single congener (hexachlorobiphenyl) and mixtures (especially Aroclor 1242) were tested. Careful controls were required since samples showed a slow decrease in PCBs with time.

Degradation by Peroxidase: Degradation of Aroclor 1242 (2 5 ppm) and hexachlorobiphenyl was tested with ligninase, PEG-ligninase, and Cytochrome $\mathrm{C}$, in the presence of $10 \% \mathrm{MeCN}$ and $0.1 \mathrm{mM} \mathrm{H} \mathrm{O}_{2}$. No evident reaction was observed. All the major peaks were reduced to a similar extent, with or without the enzyme.

Degradation by Extracts from Hyperthermophiles: Extracts from microorganisms that grow near $100^{\circ} \mathrm{C}$, including anaerobic heterotrophic archaeon, Pyrococcus furiosus, the anaerobic heterotrophic bacterium, Thermotoga maritima, and the aerophilic archaeon, Pyrobaculum aerophilum, were tested for PCB degradation with Aroclor 1242. Tested conditions included 80 and $30^{\circ} \mathrm{C}$, aerobic and anaerobic. Although some interesting variations of the major PCB peaks were observed for the extracts as compared to those of control experiments, extended degradation with addition of fresh extracts over a period of 3 days did not prove a reaction greater than the $20 \%$ estimated measurement error. These experiments were repeated using the same organisms after growth in the presence of PCB analogs. No change in the PCB fingerprint beyond the measurement error was confirmed.

\section{Further implications and future activities.}

We have discussed this effort with personnel at the Savannah River Site. We also discussed the use of a proprietary enzyme mixture from Enzyme Technologies, Inc.

From previous efforts, it appears that PCBs strongly resist attack by common redox enzymes. Future work may be conducted to seek enzymatic systems based on the consideration of redox potentials. The ionization potential of PCBs may exceed the capability of single enzymes tested previously. Further work would likely progress in the following directions: 
1. Testing enzymes from PCB-degrading microorganisms including multi-enzyme systems. Studies on in vivo microbial degradation of PCBs have revealed that the major pathway involves four specific enzymes, biphenyl dioxygenase (BphA), dihydrodiol dehydrogenase (BphB), 2,3dihydroxybiphenyl dioxygenase (BphC), and 2-hydroxyl-6-oxo-6-pheylhexa-2,4-dienoic acid hydrolase $(\mathrm{BphD})$, which catalyze sequentially the oxidative degradation of PCBs, into chlorobenzoates and 2-hydroxypenta-2,4-dienoate;

a) Developing new enzymes. An alternative to the use of modified enzymes is to utilize other, better characterized systems (such as that for TCE degradation).

b) Testing the use of multi-enzyme redox systems for a slightly simpler system (primary dechlorination of TCE which has three characterized enzymes).

c) We have demonstrated generic methods to modify enzymes to be active and soluble in organics. We should further develop modification methods to cause the enzymes to preferentially segregate into either the organic phase or the interface.

The efforts are being summarized in several publications.

\section{Relevance, Impact and Technology Transfer}

How does this new scientific knowledge focus on critical DOE environmental management problems?

This research program allows further development of nonaqueous bioremediation technologies for the treatment of DOE sites contaminated with aqueous insoluble organic compounds. Such compounds include dense nonaqueous phase liquids (DNAPL's), trichloroethylene (TCE), trichloroacetic acid (TCA), trans-dichloroethylene (DCE), diesel fuel, and polychlorinated biphenyls (PCB's). These compounds have been identified as targets for technology development in the "EM Technology Needs Database", and are contaminants at the following DOE sites: K-25 Site Plumes, ORNL WAG1, 4 and 5, Paducah Plumes, Portsmouth Plumes and X-701B Holding Pond, and the Y-12 Poplar Creek and Bear Creek Watersheds.

How will the new scientific knowledge that is generated by this project improve technologies and cleanup approaches to significantly reduce future costs, schedules, and risks and meet DOE compliance requirements?

The overall premise for this study was that bioremediation reactions typically involve recalcitrant organic materials that are not readily soluble in aqueous solution and for which most enzymes have little or no affinity. The design of biocatalysts that are active both in water and in a range of organic solvents obviates this problem. In addition, substrate specificities will depend on the nature of the solvent used. A second consideration is the stability of the biocatalyst. It must be robust and able to operate over an extended temperature range. Moreover, since various organic solvents have boiling points well above $100^{\circ} \mathrm{C}$, the ideal case would be one that utilized extremely thermostable and solvent-soluble biocatalysts. These would be able to catalyze reactions in solvents above the normal boiling point of water, and would do so in the absence of 
the destructive hydrolytic reactions of water. Such catalysts would have enormous potential not only in remediation technologies but also in diverse areas of chemical synthesis.

Thus, the objective in the research was to achieve an ideal operating condition for bioconversion, and to do this we used enzymes purified from hyperthermophiles. This recently discovered group of microorganisms thrive in volcanic vent environments at temperatures near and above $100^{\circ} \mathrm{C}$. A number of enzymes and proteins have been purified from these organisms, and many of them were obtained in the laboratory of one of the co-P.I.s. Virtually all show extreme stability when the purified forms are incubated at temperatures near $100^{\circ} \mathrm{C}$. For example, ferredoxin, which was the first protein to be purified from a hyperthermophile used for this project, was unaffected after 24 hours at $90^{\circ} \mathrm{C}$. Thus, the remarkable stability of hyperthermophilic enzymes should make them much more amenable to chemical modification with retention of catalytic activity.

To what extent does the new scientific knowledge bridge the gap between broad fundamental research that has wide-ranging applications and the timeliness to meet needs-driven applied technology development?

Key pieces of information, which can be used immediately to significantly enhance enzymatic processes, include the methods and protocols developed for putting enzymes into aqueous/ organic mixtures while maintaining their activity. Still, the technology would require smaller scale testing once a site/need is specifically chosen.

What is the project's impact on individuals, laboratories, departments, and institutions? Will results be used? If so, how will they be used, by whom, and when?

Academic and fundamental research communities will be the primary users of this information due to its basic nature. We have received requests for both electronic and hard copies of our papers. In terms of application to a DOE focus area, we hope to locate end users who are willing to support pilot implementation.

Are larger scale trials warranted? What difference has the project made? Now that the project is complete, what new capacity, equipment or expertise has been developed?

We have obtained promising results to demonstrate the proposed remediation strategy for simple systems but several fundamental questions remain to be answered. We are not ready to try complex degradation systems that would be required for highly recalcitrant compounds such as PCBs. New expertises have been developed in modifying enzymes to be more soluble and more stable in aqueous/organic mixtures.

How have the scientific capabilities of collaborating scientists been improved?

This collaborative project brought together very different yet complementary expertises from two institutions. The P.I., Dr. Davison at Oak Ridge National Laboratory, has extensive experience in bioprocess engineering for fuels, chemicals and remediation, while the co-P.I., Dr. Adams at the University of Georgia, routinely grows several hyperthermophiles in large scale culture (600 liters) and from them has purified a variety of enzymes and proteins. In essence, this project utilized the experience of both laboratories with the objective of producing a variety of solvent-soluble biocatalysts that were able to function in aqueous/organic environments for 
bioremediation reactions. The investigations into the physical and catalytic properties of chemically-modified hyperthermophilic hydrogenases and potential electron carriers as model systems for bioremediation reactions in organic solvents have increased our understanding of these systems and have spurred us to do new work in related areas. Exploring modified hydrogenase and other enzymes and how they can be utilized in remediation reactions via novel reaction pathways in organic liquids have also been beneficial.

What additional scientific or other hurdles must be overcome before the results of this project can be successfully applied to DOE Environmental Management problems?

This work is fundamental/basic and would require scale-up expertise to implement on a large scale. Several fundamental questions remain unanswered, particularly for larger multi-enzyme systems. In particular, the dechlorination of PCBs appears to be beyond the potential of s single redox enzyme. (In nature when this $\mathrm{PBC}$ dechlorination occurs, it is part of a multienzyme pathway.) Future work was proposed to focus on development of multienzyme systems for dechlorination.

Have any other government agencies or private enterprises expressed interest in the project? Please provide contact information.

\section{Project Productivity}

Did the project accomplish all of the proposed goals? Yes

Was the project on schedule? Yes (extension granted to 7/00).

Was the work plan revised? No

\section{Personnel Supported}

List professional personnel (Faculty, Post-Docs, Graduate Students, etc.) supported by and/or associated with the research effort

Brian H. Davison, Ph.D., ORNL, University of Tennessee

Erik Kaufman, Ph.D., ORNL

Michael Adams, Ph.D., Professor, Dept. of Biochemistry, University of Georgia

Ping Wang, PostDoc/ORNL, Assistant Professor, University of Akron

C. Kim, Post-Doc, University of Georgia

Kesen Ma, post-doc, University of Georgia - now at University Waterloo

Robert Weiss, University of Utah (collaborator on sequencing)

Phillip S. Brereton, Post-doc, University of Georgia

Marc F. J. M.Verhagen, Post-doc, University of Georgia - now at Allergan Corporation

Rajat Sapra, Ph.D. student, University of Georgia

Charlene A. Woodward, technician, ORNL 


\section{Publications}

P. Wang, C. A. Woodward, E. N. Kaufman, (1999) "Poly(ethylene glycol)-modified ligninase enhances pentachlorophenol biodegradation in water-solvent mixtures," Biotech. Bioeng., 64, 290-297.

P. Wang, E. N. Kaufman, C. Kim., B. H. Davison, (2000) "On the oxidation reaction of pentachlorophenol catalyzed by ligninase in the presence of hydrogen peroxide," Water Res., (submitted).

Kim, C., Woodward, C. A., Kaufman, E. N. and Adams, M. W. W. (1999) "Stability and sulfur reduction activity in organic media of the hydrogenase from the hyperthermophile Pyrococcus furiosus" Biotech. Bioeng. 65, 108-113

Ma, K. Weiss, R and Adams, M. W. W. (2000) "Characterization of hydrogenase II from the hyperthermophilic archaeon Pyrococcus furiosus and assessment of its role in sulfur reduction" J. Bacteriol.182, 1864-1871.

Ma, K. and Adams, M. W. W. (2000) Hydrogenase I and hydrogenase II from Pyrococcus furiosus" Meths. Enzymol. 330, in press.

Kim, C., Brereton, P. S., Verhagen, M. F. J. M. and Adams, M. W. W. (2000) "Ferredoxin from Pyrococcus furiosus" Meths. Enzymol. (in press).

Sapra, R. Verhagen, M. F. J. M. and Adams, M. W. W. (2000) Purification and characterization of a membrane-bound hydrogenase from the hyperthermophilic archaeon Pyrococcus furiosus" $J$. Bacteriol. 182, 3423-3428

Kim, C., Dawan, I, and Adams, M. W. W. (2000) "Properties of the ferredoxin from the hyperthermophilic archaeon Pyrococcus furiosus in organic solvents" Biotech. Bioeng. (in prep.).

\section{Interactions}

Participation/presentations at meetings, workshops, conferences, seminars, etc. We have presented platform and poster presentations at several national conferences including

Davison, B.H., Kaufman, E.N., Wang, P., Adams, M.W., Kim, C.et al. 2000. "Chemically Modified Enzymes for Remediation Reactions in Organic Media," EMSP National Workshop, Atlanta, GA USA, U.S. Dept. of Energy, Idaho Operations Office, Savannah River Operations Office, 4/24/2000-4/27/2000.

Wang, P., C.A. Woodward and E.N. Kaufman 1998. "Peg-Modified Ligninase for the Degradation of Recalcitrant Pollutants in Water-Solvent Mixtures," 216th Natl. Meet. Am. Chem. Soc., Boston, MA, Aug.23-27,1998. 
Wang, P. and B.H. Davison 1998. "Preliminary Studies of Stabilization of Enzymes for the Epoxidation of Alkenes in Nonaqueous Media," Symp. on Biotechnology for Fuels and Chemicals, Fort Collins, CO, May 2-6, 1999.

Wang, P., C.A. Woodward and E.N. Kaufman 1998. "Activation and Stabilization of Ligninase for Remediation of Polychlorinated Pollutants in Organic Solvents," Annu. Meet. Am. Inst. Chem. Eng., Miami Beach, FL, Nov.15-20,1998.

Wang, P., C.A. Woodward and E.N. Kaufman 1998. "Poly(ethylene glycol)-Modified Liginase Enhances Pentachlorophenol Biodegradation in Water-Solvent Mixtures," NSF Thermophile Workshop, Seattle, WA, May 26-29,1998.

Wang, P., C.A. Woodward and E.N. Kaufman 1997. "Modification of Enzymes for NonAqueous Catalysis: Biodegradation of Pentachlorophenol by PEG-Modified Ligninase," 20th Symp. on Biotechnology for Fuels and Chemicals, Gatlinburg, TN, May 3-7,1998.

Wang, P., C.A. Woodward and E.N. Kaufman 1997. "Modification of Enzymes for NonAqueous Catalysis: Bioremediation of Chlorinated Pollutants in Orga nic Solvents," 1st Int. Conf. on Remediation of Chlorinated and Recalcitrant Compounds, Columbus, OH, May 18-21,1998.

Kim, C. H. and M. W. W. Adams, 1997. "Enzymatic catalysis in organic solvents." The 4th National Science Foundation Hyperthermophile Symposium, San Diego, CA, May 14-19, 1997.

Adams, M. W. W. and C. H. Kim, 1997. Properties of hydrogenase from Pyrococcus furiosus" Fifth International Conference on Hydrogenases, Albertville, France, July 13-17, 1997

Kim, C. H. and M. W. W. Adams, 1998. "Catalytic and spectroscopic studies of polyethylene glycol-modified, hyperthermophilic proteins in organic solvents." The 5th Annual National Science Foundation Hyperthermophile Symposium, Seattle, WA, May 28-30, 1998

Adams, M. W. W., R. Sapra and Kim, C. H. 1998. "Sulfur reduction activity of hydrogenases." International Workshop on Hydrogenases, University of Ümea, Sweden, June 9-13, 1998

Adams, M. W. W., R. Sapra and K. Ma, 1999 "Characterization and physiological role of the membrane-bound hydrogenase of Pyrococcus furiosus" Swiss Society of Microbiology, La Chaux-de-Fonds, Switzerland, May 16-19, 1999

Kim, C. H., Dhawan, I, and M. W. W. Adams, 1999. "Properties of the ferredoxin iron-sulfur cluster from the hyperthermophilic archaeon Pyrococcus furiosus in organic solvents." The 6th Annual National Science Foundation Hyperthermophile Symposium, Athens, GA, May 19-21, 1999

\section{Collaborations.}


Co-Investigator: Dr. M. W. W. Adams, Dept. of Biochemistry, The University of Georgia, Athens, GA 30602-7229. (706)542-2060; adams@bmb.uga.edu

Other researchers: Dr. P. Wang (U. Akron); Dr. C. Kim (Postdoc, U GA),

Robert Weiss, University of Utah (collaborator on sequencing)

\section{Transitions}

None

\section{Patents}

None

\section{Future Work}

Several fundamental questions remain unanswered, particularly for larger multienzyme systems. In particular, the dechlorination of PCBs appears to be beyond the potential of s single redox enzyme. (In nature when this PBC dechlorination occurs it is part of a multienzyme pathway.) Future works was proposed to focus on development of multienzyme systems for dechlorination.

From previous efforts, it appears that PCBs strongly resist attack by common redox enzymes. Future work may be conducted to seek enzymatic systems based on the consideration of redox potentials. The ionization potential of PCBs may exceed the capability of single enzymes tested previously. Further work would likely progress in the following directions:

A. Testing enzymes from PCB-degrading microorganisms including multi-enzyme systems. Studies on in vivo microbial degradation of $\mathrm{PCBs}$ have revealed that the major pathway involves four specific enzymes, biphenyl dioxygenase (BphA), dihydrodiol dehydrogenase (BphB), 2,3-dihydroxybiphenyl dioxygenase (BphC), and 2hydroxyl-6-oxo-6-pheylhexa2,4-dienoic acid hydrolase $(\mathrm{BphD})$, which catalyze sequentially the oxidative degradation of $\mathrm{PCBs}$, into chlorobenzoates and 2-hydroxypenta-2,4-dienoate;

B. Developing new enzymes. An alternative to the use of modified enzymes is to utilize other, better characterized systems (such as that for TCE degradation).

C. Testing the use of multi-enzyme redox systems for a slightly simpler system (primary dechlorination of TCE which has three characterized enzymes).

D. We have demonstrated generic methods to modify enzymes to be active and soluble in organics. We should further develop modification methods to cause the enzymes to preferentially segregate into either the organic phase or the interface. A renewal EMSP proposal entitled "Multi-Enzyme Redox Systems for Bioremediation of Chloroorganics" was submitted; this concept has also been submitted in revised form to NSF. The proposal abstract follows: 
\{PRIVATE \}The primary objective of the current work is to gain a fundamental understanding of expression, use and regeneration of multi-step redox enzyme pathways and to modify these systems so that they will perform at optimized conditions on recalcitrant, poorly soluble, chlorinated compounds (e.g., TCE (trichloroethylene)).

The prior project emphasized the characterization of chemically-modified enzymes to attack chlorinated organics directly in an organic environment. In this project we showed that: (1) redox enzymes can work in organic environments with oxidants for the degradation of organic pollutants; (2) The use of single enzymes usually leads to intermediate products that may be of environmental concern. Experimentally, single enzymes may also be unable to attack very recalcitrant organics such as PCBs. Based on these results and the literature, we have determined that the critical stages in mineralization of recalcitrant, poorly soluble chloroorganics (i.e., PCB and TCE) generally involve reductases and oxidases acting together in reactions mediated by cofactors, typically NADH.

Accordingly, the current proposal focuses first on the assembly of a multienzyme system to attack TCE, followed by efforts to improve the system's efficiency and utility. We will assemble a three component redox enzyme system to attack TCE - an oxidase to react the $\mathrm{TCE}$, the energetic coenzyme or cofactor, $\mathrm{NADH}$, and a hydrogenase to regenerate the cofactor. Toluene monooxygenase (TMO) will be examined for the linked activity as well as stability and is known in vivo to degrade TCE into organic acids. This system will be able to directly utilize hydrogen as the reductant and energy source via hydrogenase. The hydrogenase is derived from a thermophilic source and will be optimized with respect to availability and stability. We will examine several analogues for NADH as well as tethering the cofactor to the other enzymes for retention. We will test this three-component system in an aqueous-soluble form, co-immobilized for aqueous use, and modified for organic-aqueous interfacial reaction as well as for a novel direct reaction of vapors using dry enzymes. The novelty of the proposed work lies in the breakthrough use of a combined in-situ regenerable system of reductase-cofactor-oxygenase can function with direct use of hydrogen and oxygen as the reductant and oxidant, respectively. The success of this system will alleviate bioremediation concerns of costly cosubstrates and release of genetically modified organisms.

\section{Literature Cited}

None

\section{Feedback}

None

\section{Appendices}

None 